\title{
German-Wide Analysis of the Prevalence and the Propagation Factors of the Zoonotic Dermatophyte Trichophyton benhamiae
}

\author{
Max Berlin ${ }^{1}$, Christiane Kupsch ${ }^{1}$, Lea Ritter ${ }^{1}$, Benjamin Stoelcker ${ }^{2}$, \\ Anton Heusinger ${ }^{3}$ and Yvonne Gräser ${ }^{1, *}$ \\ 1 Institute of Microbiology and Immunology, Humboldt-Universität zu Berlin and Berlin Institute of Health, \\ corporate member of Freie Universität Berlin, Universitätsmedizin Berlin, 12203 Berlin, Germany; \\ m.berlin.biotech@gmail.com (M.B.); christiane.kupsch@charite.de (C.K.); lea-ritter-2@web.de (L.R.) \\ 2 SYNLAB Vet GmbH, 86156 Augsburg, Germany; Benjamin.Stoelcker@synlab.com \\ 3 LABOKLIN GmbH \& Co. KG, 97688 Bad Kissingen, Germany; heusinger@laboklin.com \\ * Correspondence: yvonne.graeser@charite.de; Tel.: +49-(0)30-450524066
}

Received: 29 July 2020; Accepted: 31 August 2020; Published: 3 September 2020

\begin{abstract}
For about 10 years, a new variant of the pathogen Trichophyton (T.) benhamiae has appeared in Germany, characterized by a previously unobserved culture phenotype with a strong yellow reverse. A few studies suggest that this new variety is now the most common zoophilic dermatophyte in Germany. The guinea pig is the main carrier. Exact prevalence measurements are not yet available. Thus, the aim of our ongoing study was to collect data on the frequency and geographic distribution of the pathogen and its phenotypes (white and yellow) in humans and guinea pigs throughout Germany. Our former studies have already shown that animals from large breeding farms are particularly heavily affected. In contrast to this, 21 small, private breedings were sampled and husbandry conditions recorded. This placed us in a position to identify propagation factors and to give recommendations for containment. For animals from private breedings, we detected T. benhamiae with a prevalence of $55.4 \%$, which is a reduction of nearly $40 \%$ compared with animals from large breeding farms. As risk factors, we identified the type of husbandry and the contact to other breedings. Furthermore, certain animal races, like Rex guinea pigs and races with long hair in combination with curls were predestined for colonization with T. benhamiae due to their phenotypic coat characteristics. A prevalence for infections with $T$. benhamiae of $36.2 \%$ has been determined for symptomatic pet guinea pigs suspected of having dermatophytosis and is comparable to the study of Kraemer et al. showing a prevalence of $34.9 \%$ in 2009 in Germany. The prevalence in humans is stable with about 2-3\% comparing the data of 2010-2013 and 2018 in Thuringia. The new type of T. benhamiae was by far the most frequent cause in all settings.
\end{abstract}

Keywords: dermatophyte; Trichophyton benhamiae; epidemiology; prevalence; guinea pig; emerging pathogen; breeding; pets

\section{Introduction}

Since the turn of the millennium, infections with the yellow phenotype of $T$. benhamiae have increased [1]. This fungus is not only isolated from symptomatic guinea pigs, but also causes highly inflammatory infections in humans, especially children, who have had contact with these animals. Kraemer et al. reported that in approximately one quarter of the cases, humans showed clinical signs of dermatophytosis. In half the households, only children were affected [2]. In the past it was unclear where the source of the fungal infection or colonization of the guinea pigs was located. Therefore, we had systematically examined guinea pigs in pet shops for the first time [1]. Up to now, 
there have been only single case reports of infections with $T$. benhamiae among staff of pet shops. Simultaneously with ours, a Dutch study [3] was published, which showed that $16.8 \%$ of the guinea pigs from pet shops carried dermatophytes, $88 \%$ of which were T. benhamiae. In contrast, our study was able to show that more than $90 \%$ of the 59 animals examined were mainly asymptomatically colonized, that is, they carry and can transmit the pathogen. Most of the animals in the 16 pet shops in Berlin were delivered by large commercial breeders (pers. comm./animal trader). The five animals from the only private breeding were free of $T$. benhamiae. This result allowed the assumption that animals from private breeders are generally less strongly colonized with the pathogen. Therefore, guinea pigs were sampled in 21 private breeders' farms spread over Germany, and their husbandry conditions were documented. The study also served to collect data on the nationwide prevalence of $T$. benhamiae infections in humans and pet guinea pigs, as there are only limited studies available on this topic.

\section{Material and Methods}

\subsection{Study Structure}

\subsubsection{Private Breeding}

To determine the prevalence of $T$. benhamiae in breedings, 21 private farms, spread over the whole country, were visited and 381 guinea pigs, symptomatic and asymptomatic, were sampled. Besides existing symptoms indicating a possible dermatophytosis, further data on the animal and the husbandry were documented to identify possible risk factors which favor a colonization with T. benhamiae. These factors included the type of housing environment and population dynamics, measurement of prevailing humidity, race and age of the animal, as well as different coat morphologies, such as hair length and the presence of curls and other coat-free areas.

\subsubsection{Pets}

In order to analyze the frequency of $T$. benhamiae in skin infections of guinea pigs in German households, we received isolates from LABOKLIN (Bad Kissingen, Germany) and SYNLAB Vet (Augsburg, Germany) over a period of 10 months, from February to November 2019, which tested positive for dermatophytes in routine diagnostics with in-house PCR methods. In our laboratory, the exact species differentiation was then carried out. In total, we received 435 isolates, of which 236 isolates (192 cultures, 44 DNA isolates) were from LABOKLIN and 199 isolates (DNA isolates only) from SYNLAB Vet. Residual samples of the DNA isolates were stored at $-20{ }^{\circ} \mathrm{C}$ for later analysis. Culture plates were stored at $4{ }^{\circ} \mathrm{C}$.

\subsection{Questionnaires}

In order to determine the prevalence of $T$. benhamiae in humans and animals, questionnaires were sent to practicing veterinarians and dermatologists throughout Germany to obtain the number of cases for the year 2018 .

About 900 dermatological practices and clinics as well as about 200 veterinary dermatologists were contacted directly via e-mail. A total of 3500 veterinarians were informed via the newsletter of the German Veterinary Medical Society (DVG). In addition, an advertisement with a link to the questionnaire was published in the monthly members' magazine of the Bundesverband Praktizierender Tierärzte e.V. as well as in an issue of the Deutsches Tierärzteblatt. For the year 2018, the total number of examined patients and infections with T. benhamiae were questioned. For the T. benhamiae diagnoses, it was asked whether these were determined by molecular biological or cultural diagnostics. For cultural findings, the color of the culture could be additionally indicated. Dermatologists had the additional possibility to indicate the location of the human infection and whether guinea pigs were kept in the patient's household (for the questionnaire, see Supplementary Materials). Veterinarians should additionally indicate the number of T. mentagrophytes infections in guinea pigs and the number of cases 
in which a zoonotic transmission to the keeper was known. If other dermatophytes have been detected on guinea pigs, the species and number could be noted additionally.

\subsection{Diagnostics}

\subsubsection{Sampling of Guinea Pigs}

For the sampling, the animals were brushed intensively with a toothbrush according to MacKenzie, concentrated mainly on the back (dorsal), flanks (lateral), and head area. The use of interchangeable toothbrushes (yaweco, Bernau im Schwarzwald, Germany, medium, cat\# 101020) allowed a quick transfer of the head brushes into a sterile $50 \mathrm{~mL}$ reaction tube (Falcon/Corning Science, New York, NY, USA).

\subsubsection{Culture}

Clinical samples were cultivated on two different culture media, on Sabouraud agar with gentamicin and chloramphenicol and on Sabouraud agar with gentamicin, chloramphenicol, and cyclohexamide. Cultivation was at $28{ }^{\circ} \mathrm{C}$ for at least 3 weeks. If no growth was visible after this time, the sample was considered negative.

\subsubsection{DNA Extraction}

For the subsequent DNA isolation from clinical samples, the Qiagen Blood \& Tissue Kit (Qiagen, Hilden, Germany, cat\# 69506) was used according to the manufacturer's protocol with slight modifications. The brush was vortexed with $2 \mathrm{~mL} 0.9 \%$ sterile $\mathrm{NaCl}$ solution for $10 \mathrm{~s}$. The $\mathrm{NaCl}$ solution was then transferred to a $2 \mathrm{~mL}$ reaction tube and centrifuged for $5 \mathrm{~min}$ at $10,000 \times \mathrm{g}$. The resulting pellet was resuspended in $230 \mu \mathrm{L}$ ATL buffer (Qiagen, Hilden, Germany, cat\# 19076) and, after the addition of $20 \mu \mathrm{L}$ protein kinase K (Qiagen, Hilden, Germany, cat\# 19131), was digested overnight at

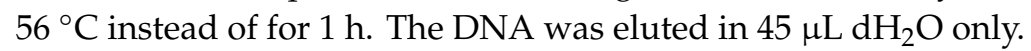

Cultures: A single fungal colony was taken from grown culture and added to $60 \mu \mathrm{L}$ lysis buffer (QuickExtract ${ }^{\mathrm{TM}}$ Plant DNA Extraction Solution, Lucigen, Middleton, Wisconsin, USA, cat\# QEP 70750). An incubation of $1 \mathrm{~h}$ at $65^{\circ} \mathrm{C}$ followed. After the lysis was stopped at $98^{\circ} \mathrm{C}$ for $2 \mathrm{~min}$, the lysate was used directly in the PCR.

\subsection{PCR Method}

To differentiate between variants of T. behamiae, a sequence region in the ITS region was chosen. The following primer sequences $\left(5^{\prime} 3^{\prime}\right)$ were used:

yellow variant: 5'-CGATAGGAATCAACGTTCCATC/5'-CCCCGAAAGAGGAGGT (for/rev) white variant: 5'-GATAGGGACCAACGTTCCG/5'-CCCGAAAGAGGGGGC (for/rev)

The specificity of the primer pair was checked using NCBI blast option and DNA of close-related zoophilic species, for example, T. erinacei and T. verrucosum.

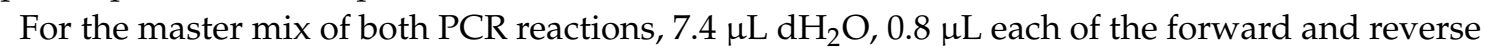
primer (50 pmol), and $10 \mu \mathrm{L}$ Biozym Red HS Taq Master Mix (Biozym Scientific, Hessisch Oldendorf, Germany, cat\# 331126) were used for each reaction ( $20 \mu \mathrm{L}$ volume). The PCR started with an initial 3 min denaturation at $95^{\circ} \mathrm{C}$, followed by 40 cycles consisting of $15 \mathrm{~s}$ denaturation $\left(95^{\circ} \mathrm{C}\right), 15 \mathrm{~s}$ annealing $\left(58^{\circ} \mathrm{C}\right)$, and $20 \mathrm{~s}$ elongation $\left(72{ }^{\circ} \mathrm{C}\right)$. The final elongation step was $60 \mathrm{~s}$.

If neither of the two variants was detected, a pan-dermatophyte-specific PCR and external sequencing (LGC Genomics GmbH, Berlin, Germany) followed for species differentiation. For this PCR, the ITS5 primer [4] was used in combination with a pan-dermatophyte-specific reverse primer [5] to amplify the entire ITS1 region. The annealing temperature was $56^{\circ} \mathrm{C}$. If it was not possible to identify the species by sequencing, due to the presence of more than one dermatophyte species, a commercial 
test system (EUROArray Dermatomycosis, EUROIMMUN, Lübeck, Germany, cat\# MN 2850-1005) was used.

\subsection{Statistics}

The statistical analysis was performed on SPSS (IBM SPSS Statistics 23). Ratio-scaled characteristics, such as the age of the animals, were tested for significance using Mann-Whitney U-test, and nominally scaled characteristics were tested for significance using chi-square tests. A $p$-value of $<0.05$ was considered significant.

Participation in the study was voluntary, free of charge, and all data were anonymized prior to analysis.

\section{Results}

\subsection{Prevalence of Farm Animals}

Nationwide (except for Baden-Württemberg, Thuringia, and Saarland), 21 breedings with a total of 381 guinea pigs were sampled (Figure 1). The herd sizes varied between 7 and 100 animals. Samples were taken from twenty animals per breed. In herds with less than 20 animals, all of them were sampled. On $262(68.8 \%)$ of the sampled animals, a dermatophyte was detected (Figure 2). With $55.4 \%$, T. benhamiae was significantly the most frequently detected dermatophyte $(p=0.000325$, Figure 2). No conclusions could be drawn either on the geographical origin or on differences in the geographical spread of $T$. benhamiae. In our survey, $T$. benhamiae was constantly distributed over the German territory. No regional hotspot and no index case were identified.

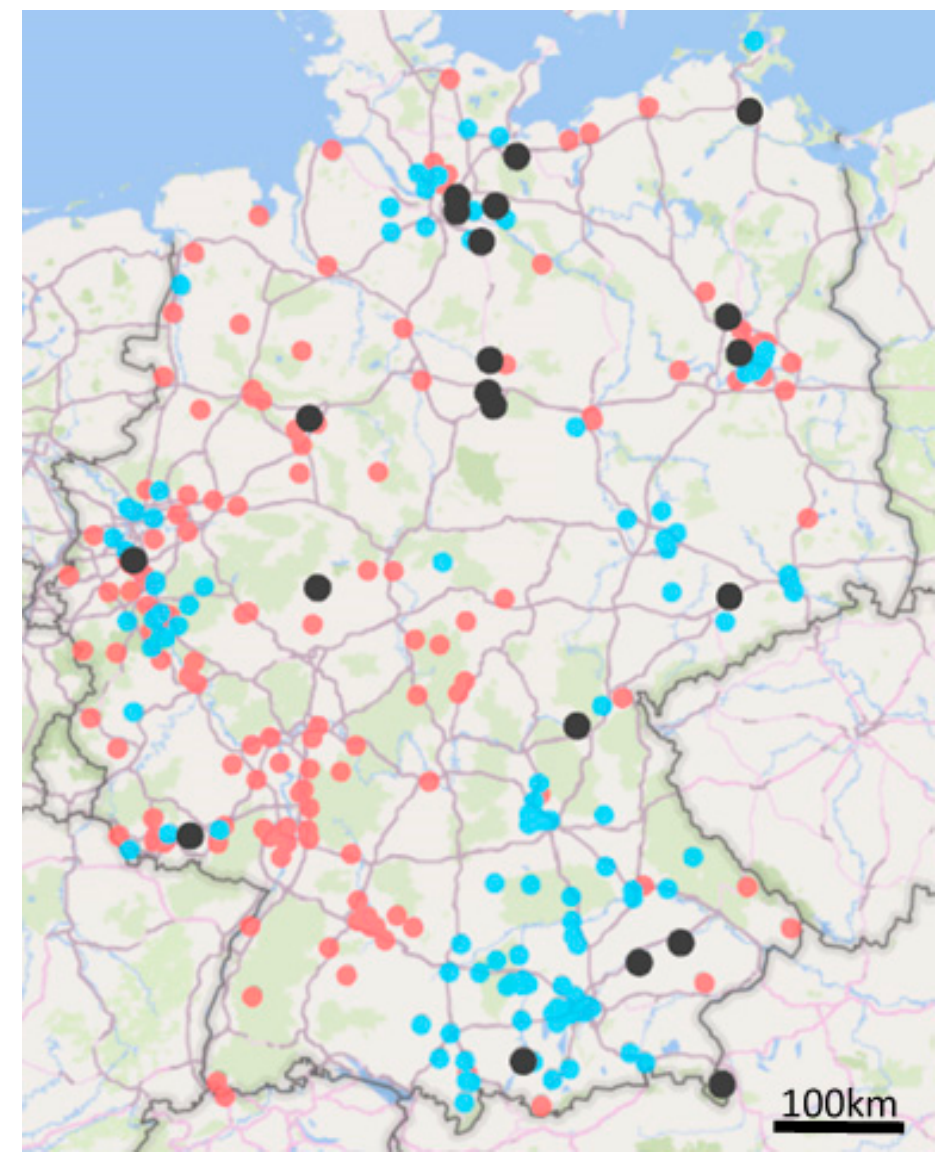

Figure 1. Sample origin, black—private breedings, blue-SYNLAB Vet, red-LABOKLIN. 


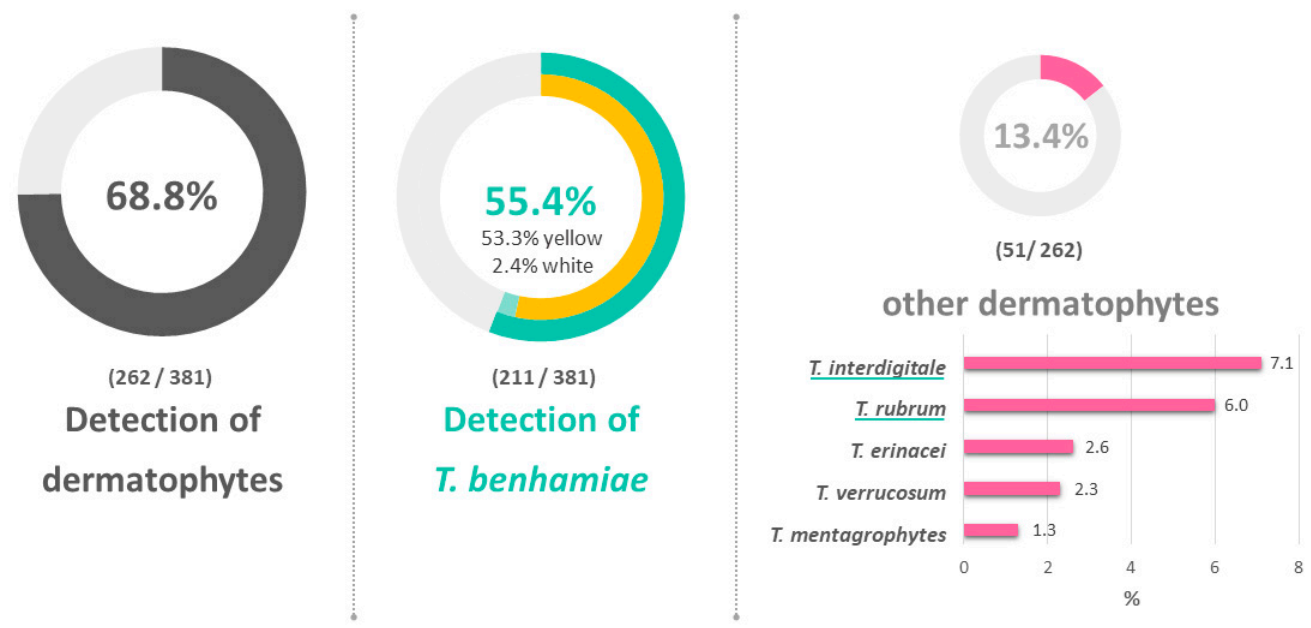

Figure 2. Detected dermatophytes on breeding guinea pigs.

In 51 animals (13.4\%) of other species, mostly anthropophilic dermatophytes such as T. interdigitale and T. rubrum were detected (Figure 2, Table 1). In 28 animals (7.3\%), symptoms of dermatophytosis were documented. In all these cases, the yellow phenotype of $T$. benhamiae was isolated. The majority of the animals, $92.7 \%$, were asymptomatically colonized, that is, carriers of the pathogens. On nine animals $(2.4 \%)$, the white phenotype of $T$. benhamiae was detected. Fourteen mixed infections were observed. The most frequent were the combinations T. interdigitale and T. rubrum (6/14) or T. interdigitale and T. verrucosum $(4 / 14)$.

Table 1. Detection of dermatophytes on breeding guinea pigs $(* 1$-both types were present in one animal $/ * 2$-partly mixed infections).

\begin{tabular}{cc}
\hline Species & Number of Samples (Percent) \\
\hline Negative & $119 / 381(31.2 \%)$ \\
Dermatophytes positive & $262 / 381(68.8 \%)$ \\
T. benhamiae (yellow) $* 1$ & $203 / 381(53.3 \%)$ \\
T. benhamiae (white) & $9 / 381(2.4 \%)$ \\
Other Dermatophytes $* 2$ & $51 / 262(13.4 \%)$ \\
T. interdigitale & $27 / 262(7.1 \%)$ \\
T. rubrum & $23 / 262(6.0 \%)$ \\
T. erinacei & $10 / 262(2.6 \%)$ \\
T. verrucosum/eriotrephon & $6 / 262(2.3 \%)$ \\
T. mentagrophytes & $5 / 262(1.3 \%)$ \\
\hline
\end{tabular}

\subsection{Influence of Risk Factors}

In order to identify risk factors that promote colonization or infection with $T$. benhamiae, possible factors related to the animal (sex and race) and the husbandry conditions (population dynamics and location) were documented during sampling (Table 2). 
Table 2. Description of possible risk factors, their observed frequency, and their influence on the detection of T. benhamiae (SH-shorthair). ${ }^{*}$ Comparison between all animal races.

\begin{tabular}{|c|c|c|c|c|}
\hline Factor & Characteristics & $\begin{array}{c}\text { Frequency }(n) \\
(\%)\end{array}$ & $\begin{array}{l}\text { T. benhamiae } \\
\text { Detection (\%) }\end{array}$ & $p$-Value \\
\hline \multirow{4}{*}{ Sex } & Female & $259(20.2)$ & 55.2 & \multirow{3}{*}{0.074} \\
\hline & Male & $77(68.0)$ & 49.4 & \\
\hline & Castrated & $45(11.8)$ & 66.7 & \\
\hline & Male + castrated & $122(32.0)$ & 55.7 & 0.506 \\
\hline \multirow{6}{*}{ Animal race/Pheno type } & Long hair + curls & $50(13.1)$ & 74.0 & \multirow{6}{*}{$0.00002 *$} \\
\hline & Long hair - curls & $39(10.2)$ & 30.8 & \\
\hline & Teddy & $90(23.6)$ & 51.1 & \\
\hline & $\operatorname{Rex}$ & 69 (18.1) & 68.1 & \\
\hline & Rosette (SH + curls) & $69(18.1)$ & 62.3 & \\
\hline & Smooth hair & $64(16.8)$ & 40.6 & \\
\hline \multirow{3}{*}{ Husbandry condition } & Outdoor & $114(29.9)$ & 51.8 & \multirow{3}{*}{0.011} \\
\hline & Cold barn & $133(34.9)$ & 46.6 & \\
\hline & Indoor & $134(35.2)$ & 67.2 & \\
\hline \multirow{2}{*}{ Stock Dynamics } & Static (autarkic) & $202(53.0)$ & 37.1 & \multirow{2}{*}{$1.19 \times 10^{-14}$} \\
\hline & Dynamic & $179(47.0)$ & 76.0 & \\
\hline \multirow{2}{*}{ Coat length } & short & $300(78.7)$ & 56.3 & \multirow{2}{*}{0.276} \\
\hline & long & $81(21.3)$ & 51.9 & \\
\hline \multirow{2}{*}{ Curls } & Ye & $118(31.0)$ & 68.6 & \multirow{2}{*}{0.000328} \\
\hline & No & $263(69.0)$ & 49.4 & \\
\hline
\end{tabular}

Castrated males were slightly more strongly colonized than uncastrated males (66.7\% vs. $49.4 \%)$. If no differentiation was made between males, no significant differences $(p=0.506)$ between the sexes were found. In advance, different races were classified on the basis of morphological aspects. In longhair races, only the presence/absence of curls was distinguished. In the short-haired races, a distinction was made between Rex, Teddy, Rosette, and smooth-haired guinea pigs (Figure 3). Significantly ( $p=0.00002)$ more frequently, longhaired races with curls $(74.0 \%)$ and Rex guinea pigs $(68.1 \%)$ were colonized with $T$. benhamiae. Smooth-haired guinea pigs were among the least affected. The influence of hairy curls, that is, the presence of fur-free areas due to a circular growth direction, was again examined in detail. Animals that were carriers of one or more curls showed significantly $(p=0.000328)$ more frequent colonization with $T$. benhamiae than animals without this feature (68.6\% vs. $49.4 \%)$. If only short and long fur was differentiated, no significant difference was found. The husbandry system was differentiated between indoor husbandry (closed, heatable rooms, $22{ }^{\circ} \mathrm{C}$ on average, recorded relative humidity 58-66\%), outdoor husbandry (open barn/open-air enclosure, temperature and relative humidity depending on weather) and cold barn management (open barn but protected from wind and weather, temperature and relative humidity depending on weather). Animals living in cold housing were the least affected (46.6\%). Indoor animals were significantly $(p=0.011)$ more affected $(76.2 \%)$. Finally, a classification of the breeds was made based on their stock dynamics. Dynamic breeding differs from static breeding by frequent new arrivals and departures. With the latter, the animal population is only subject to a few changes. These work with little contact to animals from other breeds. Animals from dynamic breeding were significantly $\left(p=1.19 \times 10^{-14}\right)$ more strongly infected with $T$. benhamiae than animals from autarkic breeding $(76.0 \%$ vs. $37.1 \%)$.

Breeders and owners reported very often symptomatic infections on young animals at the age of 1 to 3 months, as well as on very old animals ( $>5$ years). Neither the sampled breeding animals nor the data from symptomatic pets showed a significant influence of age on the prevalence of $T$. benhamiae (Mann-Whitney test $p=0.934$ ). The age distribution (as average and median) of all animals and of the mostly asymptomatic breeding and symptomatic pets shows no relevant differences (Table 3). Only the median for symptomatic pets indicates that mainly younger guinea pigs were infected with the pathogen. 

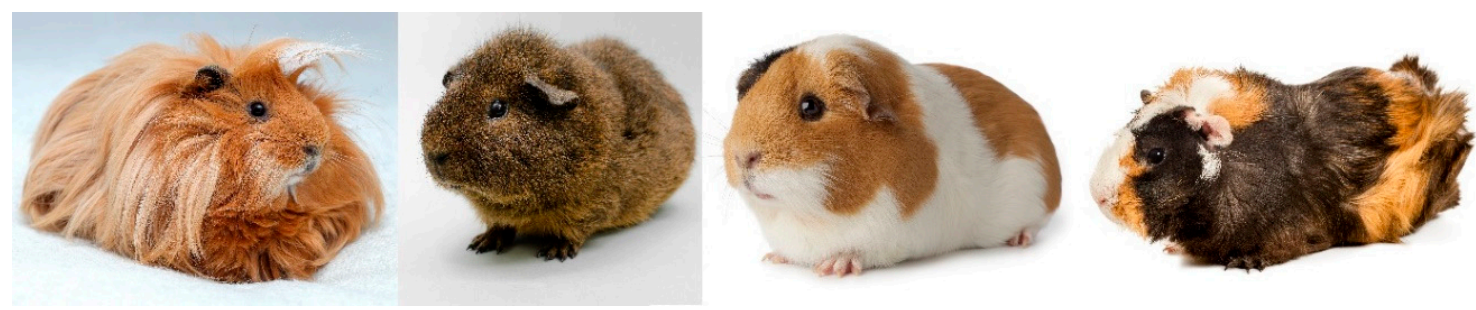

Figure 3. Guinea pig races with their different fur forms: (from left to right) longhaired guinea pigs, Rex guinea pigs, smooth-haired guinea pigs, curly-coated guinea pig (shutterstock.com/free-license images).

Table 3. Age distribution among the sampled animals.

\begin{tabular}{ccc}
\hline Age & $\begin{array}{c}\text { Average } \\
\text { (Months) }\end{array}$ & $\begin{array}{c}\text { Median } \\
\text { (Months) }\end{array}$ \\
\hline of all animals (breedings) & 15.8 & 12 \\
of animals positive for T. benhamiae (breedings) * & 15.7 & 12 \\
of symptomatic pets (laboratory data) & 17.5 & 6 \\
\hline * Asymptotic significance (Mann-Whitney test) & $=0.934$ not significant.
\end{tabular}

* Asymptotic significance (Mann-Whitney test) $=0.934$ not significant.

\subsection{Prevalence of Symptomatic Pets}

To investigate the incidence of dermatophytosis caused by T. benhamiae in guinea pigs, which are usually kept in pairs in the household, we received residual clinical samples with suspected infection from two large diagnostic laboratories, SYNLAB VET and LABOKLIN. With the support of the two large laboratories, we were able to cover samples from a wide area of Germany (Figure 1). Over a period of 10 months, February to November 2019, we differentiated 382 samples at species level. In total, the two laboratories received 9636 samples from guinea pigs during this period, of which $1035(10.7 \%)$ were submitted with suspected dermatophytosis (Table 4). Of the 382 dermatophyte infections diagnosed, 375 (98.2\%) were caused by T. benhamiae. In seven cases, other dermatophyte species were identified (Table 4). Using the PCR developed in this study, 357 (95.2\%) T. benhamiae isolates were differentiated as yellow and $18(4.8 \%)$ as white phenotype. Relative to the number of all guinea pig isolates examined in the two laboratories during this period, the prevalence of infection with T. benhamiae was $3.9 \%$. From the suspected cases of dermatophytosis, $36.2 \%$ of the animals showed an infection with $T$. benhamiae.

Table 4. Detection of dermatophytes in guinea pig samples from SYNLAB VET and LABOKLIN, February-November 2019.

\begin{tabular}{cc}
\hline Samples Analyzed & Number \\
\hline Total & 9636 \\
\hline with suspected mycosis & 1035 \\
\hline Tested positive for dermatophytes & 382 \\
\hline T. benhamiae & 375 \\
\hline T. benhamiae (yellow) & 357 \\
\hline
\end{tabular}


Table 4. Cont.

\begin{tabular}{cc}
\hline Samples Analyzed & Number \\
\hline T. benhamiae (white) & 18 \\
\hline T. rubrum & 3 \\
\hline Microsporum (M.) canis & 1 \\
\hline T. mentagrophytes & 1 \\
\hline T. spp. & 1 \\
\hline T. interdigitale & 1 \\
\hline
\end{tabular}

\subsection{Prevalence of Human T. benhamiae Infections}

In order to record the prevalence of T. benhamiae infections in humans, questionnaires were sent to dermatological practices and clinics all over Germany and microbiological laboratories were contacted. A total of 51,238 patients could be registered who presented themselves in 13 dermatological practices in 2018 or whose samples were analyzed in 3 microbiological laboratories (Table 5). Using culture or molecular biological methods, in total 163 infections with $T$. benhamiae were detected, resulting in a prevalence of $0.3 \%$. Among the 16 participants was the laboratory for medical microbiology in Mölbis (district of Leipzig), which has expertise in the diagnosis of skin fungal infections. Of the 163 infections with T. benhamiae, 124 were diagnosed with PCR methods in this laboratory alone, and 6044 patients were examined there during this period. The prevalence calculated from these data is $2.05 \%$ and thus about seven times higher compared with all laboratories $(0.3 \%)$. Animal contact is described in $39(31.5 \%)$ of the 124 patients who tested positive, and in $32(25.8 \%)$ of them, specifically with a guinea pig.

Table 5. Evaluation of questionnaires on recorded human T. benhamiae infections, 2018.

\begin{tabular}{cc}
\hline Patients Recorded & Number \\
\hline Total & 51,238 \\
Number of T. benhamiae infections & 163 \\
of which culture diagnosed & 126 \\
thereof diagnosed by PCR & 145 \\
thereof contact with guinea pigs known & $32 / 163$ \\
\hline
\end{tabular}

\section{Discussion}

The massive spread of T. benhamiae in the last two decades is related to the parallel emergence of a new phenotype, which is characterized by a yellow instead of a white-colored culture [6]. Our study shows that in fact the white phenotype was only isolated from $2.4 \%$ and $4.8 \%$ of breeding guinea pigs and pets, respectively.

Furthermore, our study investigated for the first time the distribution of T. benhamiae in private breedings. With $55.4 \%$, the prevalence of the pathogen was significantly lower than in animals from commercial animal wholesale. In a study in 2016, we were able to detect T. benhamiae on guinea pigs in Berlin pet shops, which usually came from non-animal welfare-compliant large breedings, with a prevalence of $93 \%$ [1]. In contrast, a study by Dutch colleagues at the same time was able to show that only $16.8 \%$ of guinea pigs from pet shops carried a dermatophyte, mostly T. benhamiae; however, no information on the origin of the animals was provided [3]. The same applies to a study from France in 2016, in which T. benhamiae was detected on 34 of the 51 examined guinea pigs (67\%) in three pet shops in Nancy [7]. In a Danish study, T. benhamiae was detected on the guinea pigs offered in $41 \%$ of the pet shops investigated in Copenhagen. In this case, contaminated pet shops belonged exclusively to the group of shops that purchased their animals from external sources (animal traders) and had not 
bred the animals themselves [8]. For these three studies, in contrast to our study, the culture was used as an initial diagnostic procedure, which is known to have only about $70 \%$ of the sensitivity of PCR [9].

Poor hygienic conditions and exposition to carriers are known to promote the transmission and spread of the pathogen in the animal stock and thus have a negative effect on the constitution of the animals [10]. This leads increasingly to dermatophytosis. The probability of acquiring an infected animal is approximately $50 \%$ lower in private breeding than in a pet shop that purchases animals from animal wholesalers.

Nevertheless, even a husbandry which is oriented towards the needs of the animal seems not to be able to prevent an infection of the stock with $T$. benhamiae.

The aim of the study was also to identify risk factors that influence colonization. Animals from purely indoor housing systems were significantly more likely to be colonized with the pathogen than those from outdoor and cold housing systems. Reasons for this may be the better air exchange and the associated, weather-dependent, lower air humidity.

In addition, breedings that were subject to high stock dynamics, that is, which had many new arrivals from other husbandry systems, were particularly exposed to high levels of contamination. The contact to other breeds, be it through exchange of breeding animals or at meetings (e.g., at breeding shows), could be identified as a further factor for the introduction and spread of T. benhamiae. Future strategies for containment and prevention must also start here.

Not only the housing conditions but also intrinsic factors of the guinea pigs influenced the probability of acquiring an infection. Guinea pigs with smooth hairs and with very long fur without curls were slightly less exposed to the pathogen than those with hairs that were not attached but standing up. In the former, the coat morphology means that the skin surface is rarely exposed, that is, better protected from damaging influences (trauma, lesions, UV light). In the latter, the so-called Rex and Teddy guinea pigs, the coat morphology contributes to the often dry, scaly skin (pers. comm./breeders). The influence on races with partially unprotected skin is supported by the significantly more frequent detection of the pathogen in animals with curls in our study.

A significant influence of gender on the colonization with $T$. benhamiae was not determined in our study either. This result is consistent with other studies [10,11]. Bucks (unneutered males) usually sit alone or together with a neuter and have contact with females only for mating. This reduces the risk of infection, which would explain the slightly reduced stress on these animals. Castrated males usually sit together with females in larger groups. Here, our data show a slightly increased burden. However, this effect is cancelled out if we do not differentiate between bucks and castrates in males.

There was no significant influence of age on infection/colonization with T. benhamiae in our study, confirming the study of Drouot et al. [11]. However, the median age of symptomatic pets was 6 months lower than the median of all animals ( 6 instead of 12 months). Kraemer et al. [12] and Vangeel et al. [13] reported that affected animals were significantly younger than those with negative culture results as well as the healthy animals of a control population. In 2012, the group around Kraemer et al. [12] mentioned that $40 \%$ of infected guinea pigs were younger than 5 months and calculated a median of 6 months for their cohort. This is consistent with the observations of breeders and veterinarians, who increasingly report symptomatic mycoses, especially in guinea pigs in the first 3 months of life, which are suspected to be associated with an immune deficiency after weaning (4-6 weeks of life) (pers. comm./breeders, veterinarians). The observed shift in the median of symptomatic animals may be an indication.

Besides T. benhamiae, other dermatophytes were detected in $13.4 \%$ of the examined animals, mainly two anthropophilic species, T. interdigitale and T. rubrum, whose origin was most likely the breeders themselves. Kraemer et al. [12] report further dermatophytes isolated in guinea pigs such as M. canis, M. audouinii, and T. rubrum. In addition, other studies isolated M. canis in small numbers in Germany [14] and New Zealand [15]. These results, consistent with the present study, show that dermatophytes other than the main pathogen are only infrequently isolated from guinea pigs. 
For symptomatic pet guinea pigs suspected of having dermatophytosis, we determined a prevalence of $36.2 \%$ for T. benhamiae. Kraemer et al. [12] reported a prevalence of $34.9 \%$ for T. mentagrophytes (old nomenclature) in a comparable survey for the year 2009. The authors noted that a morphological differentiation within the T. benhamiae species complex was not possible, but assumed that "the Arthroderma (A.) benhamiae complex is associated with most dermatophytoses in Guinea pigs and rabbits" [12]. Considering the very low prevalence of T. mentagrophytes of $1.3 \%$ for asymptomatic and $0.3 \%$ for symptomatic guinea pigs in our study, it is assumed that the majority in Kraemer's study was due to T. benhamiae. Based on this assumption, there was only a slight increase $(1.3 \%)$ of the prevalence within 10 years in Germany. An Italian study reported a lower prevalence of $24.7 \%$ in pet guinea pigs in 2015 (23/93) [16]. In 95.2\% of the cases, the yellow type of T. benhamiae was isolated.

For human infections caused by $T$. benhamiae, we computed a prevalence of only $0.3 \%$ from the results of the questionnaires we sent out. However, we assume that the number of unreported cases is significantly higher due to problems with the species identification, which is difficult, as T. benhamiae was described under different names during the last decades, like Arthroderma benhamiae as teleomorph of T. mentagrophytes [17] or T. mentagrophytes var. procellae [18]. This assumption is supported by the data provided by the Laboratory for Medical Microbiology in Mölbis/Leipzig during the present study, revealing a prevalence of $T$. benhamiae in 2018 of $2.05 \%$. The same laboratory published a similar prevalence of 2.9\% between March 2010 and March 2013 [6]. The authors concluded that T. benhamiae is currently more common than M. canis, at least in some regions of Germany. This trend seems to continue. In Strasbourg, a similar trend was observed: T. benhamiae was temporarily more frequent than M. canis in at least three years $(2011,2012,2014)$ of the 9 year survey [19]. These authors were also able to establish a connection to a pet guinea pig in the household in $75 \%$ of the T. benhamiae cases, of which, however, only $25.9 \%$ showed symptoms of dermatophytosis. Silent carriers of $T$. benhamiae represented a major part $(86.7 \%)$ of the animals in our study, too.

\section{Concluding Remarks}

The zoonotic potential of guinea pigs for transmitting a dermatophyte should not be underestimated. Therefore, we recommend buying a guinea pig from private breeding rather than from wholesalers because the probability of acquiring an infected animal is approximately $50 \%$ lower. In any case, diagnostic screening of guinea pigs ideally by molecular methods is recommended. The transmission of T. benhamiae can be reduced by outdoor and cold housing systems, static populations, and intrinsic factors like the coat morphology (smooth hair, no curls). In the last 10 years in Germany, the frequency of $T$. benhamiae infections in pet guinea pigs did not change. In Thuringia, a part of Germany, however, the frequency of human T. benhamiae infections is stable but on a high level compared with 2010/13. Breeders and animal owners must therefore be made aware of the zoonotic risk and how to minimize it. Therefore, we have drawn up a guideline (see Supplementary Materials).

Supplementary Materials: The following are available online at http://www.mdpi.com/2309-608X/6/3/0161/s1. S1 Questionnaire sent to dermatologists and microbiological labs. S2 Guideline for owners and breeders of guinea pigs.

Author Contributions: All authors have read and agreed to the published version of the manuscript. M.B. performed the study and prepared the manuscript. C.K. applied for research funding. L.R. performed the PCR experiments. B.S. and A.H. collected the samples from household guinea pigs. Y.G. applied for research funding and prepared the manuscript.

Funding: Was provided by the Nationale Forschungsplattform für Zoonosen, grant 01KI1805.

Acknowledgments: We thank the breeders who made the study possible with their participation, the participants in the survey and the Mölbis laboratory for the data provided. We acknowledge support from the German Research Foundation (DFG) and the Open Access Publication Funds of Charité - Universitätsmedizin Berlin.

Conflicts of Interest: The authors declare no conflict of interest. The funders had no role in the design of the study; in the collection, analyses, or interpretation of data; in the writing of the manuscript, or in the decision to publish the results. 


\section{References}

1. Kupsch, C.; Berlin, M.; Gräser, Y. Dermatophyten und Meerschweinchen Dermophytes and guinea pigs. Der Hautarzt 2017, 68, 827-830. [CrossRef]

2. Kraemer, A.; Hein, J.; Heusinger, A.; Mueller, R.S. Clinical signs, therapy and zoonotic risk of pet guinea pigs with dermatophytosis. Mycoses 2012, 56, 168-172. [CrossRef] [PubMed]

3. Overgaauw, P.; Avermaete, K.; Mertens, C.; Meijer, M.; Schoemaker, N. Prevalence and zoonotic risks of Trichophyton mentagrophytes and Cheyletiella spp. in guinea pigs and rabbits in Dutch pet shops. Vet. Microbiol. 2017, 205, 106-109. [CrossRef] [PubMed]

4. White, T.J.; Bruns, T.; Lee, S.; Taylor, J. Amplification and Direct Sequencing of Fungal Ribosomal RNA Genes for Phylogenetics. In PCR Protocols; Elsevier: Amsterdam, The Netherlands, 1990; pp. 315-322.

5. Kupsch, C.; Czaika, V.A.; Deutsch, C.; Graeser, Y. Trichophyton mentagrophytes-A new genotype of zoophilic dermatophyte causes sexually transmitted infections. J. Dtsch. Dermatol. Ges. 2019, 17, 493-501. [CrossRef] [PubMed]

6. Uhrlaß, S.; Krüger, C.; Nenoff, P. Microsporum canis: Current data on the prevalence of the zoophilic dermatophyte in central Germany. Hautarzt 2015, 66, 855. [CrossRef] [PubMed]

7. Bloch, M.; Cavignaux, R.; Debourgogne, A.; Dorin, J.; Machouart, M.; Contet-Audonneau, N. From guinea pig to man: Tinea outbreak due to Trichophyton mentagrophytes var. porcellae in pet shops in Nancy (France). J. Mycol. Med. 2016, 26, 227-232. [CrossRef] [PubMed]

8. Tekin, H.G.; Sigsgaard, V.; Zachariae, C.; Hare, R.K.; Arendrup, M.C.; Saunte, D. Would you like to purchase a rodent with dermatophytes? Mycoses 2019, 62, 584-587. [CrossRef] [PubMed]

9. Kupsch, C.; Ohst, T.; Pankewitz, F.; Nenoff, P.; Uhrlaß, S.; Winter, I.; Gräser, Y. The agony of choice in dermatophyte diagnostics-Performance of different molecular tests and culture in the detection of Trichophyton rubrum and Trichophyton interdigitale. Clin. Microbiol. Infect. 2016, 22, 735.e11-735.e17. [CrossRef] [PubMed]

10. Hein, J. Dermatophytose bei Kaninnchen und Meerschweinchen-Ein Update einer Zoonose. Kleintierpraxis 2016, 61, 675-688.

11. Drouot, S.; Mignon, B.; Fratti, M.; Roosje, P.; Monod, M. Pets as the main source of two zoonotic species of the Trichophyton mentagrophytes complex in Switzerland, Arthroderma vanbreuseghemii and Arthroderma benhamiae. Vet. Dermatol. 2009, 20, 13-18. [CrossRef] [PubMed]

12. Kraemer, A.; Mueller, R.S.; Werckenthin, C.; Straubinger, R.K.; Hein, J. Dermatophytes in pet Guinea pigs and rabbits. Vet. Microbiol. 2012, 157, 208-213. [CrossRef] [PubMed]

13. Vangeel, I.; Pasmans, F.; Vanrobaeys, M.; De Herdt, P.; Haesebrouck, F. Prevalence of dermatophytes in asymptomatic guinea pigs and rabbits. Vet. Rec. 2000, 146, 440-441. [CrossRef]

14. Weiss, R.; Böhm, K.H.; Mumme, J.; Nicklas, W. 13 Years of veterinary mycological routine diagnostics. Isolation of dermatophytes in the years 1965-1977. Sabouraudia 1979, 17, 345-353. [PubMed]

15. Smith, J.M.B.; Rush-Munro, F.M.; McCarthy, M. Animals as a Reservoir of Human Ringworm in New Zealand. Australas. J. Dermatol. 1969, 10, 169-182. [CrossRef] [PubMed]

16. D'Ovidio, D.; Santoro, D. Survey of Zoonotic Dermatoses in Client-Owned Exotic Pet Mammals in Southern Italy. Zoonoses Public Health 2014, 62, 100-104. [CrossRef] [PubMed]

17. de Hoog, G.S.; Guarro, J.; Gené, J.; Ahmed, S.; Al-Hatmi, A.M.S.; Figueras, M.J.; Vitale, R.G. Atlas of Clinical Fungi 2019, 3rd ed.; Reus: Utrecht, The Netherlands, 2019.

18. Contet-Andonneau, N.; Leyer, C. Émergence d'un dermatophyte transmis par le cochon d'Inde et proche de Trichophyton mentagrophytes var. erinacei: T. mentagrophytes var. porcellae. J. Mycol. Med. 2010, 2, 321-325. [CrossRef]

19. Sabou, M.; Denis, J.; Boulanger, N.; Forouzanfar, F.; Glatz, I.; Lipsker, D.; Poirier, P.; Candolfi, E.; Letscher-Bru, V. Molecular identification of Trichophyton benhamiae in Strasbourg, France: A 9-year retrospective study. Med. Mycol. 2017, 56, 723-734. [CrossRef] [PubMed]

(C) 2020 by the authors. Licensee MDPI, Basel, Switzerland. This article is an open access article distributed under the terms and conditions of the Creative Commons Attribution (CC BY) license (http://creativecommons.org/licenses/by/4.0/). 